\title{
P1: Selbstmanagementförderung Pflegebedürftiger und ihrer pflegenden Angehörigen zur Vorbereitung auf die häusliche Selbstversorgung - ein neues Aufgabenfeld in der stationären Pflege
}

\author{
Anke Menzel-Begemann • Britta Klünder • \\ Doris Schaeffer
}

Online puliziert: 23. Oktober 2013

(C) Springer-Verlag Wien 2013

Einleitung: Für Erkrankte und Angehörige ist die Rückkehr in die häusliche (Selbst-) Versorgung nach stationärem Aufenthalt ein mit Unsicherheiten behafteter Schritt. Denn die Versorgungssituation verändert sich grundlegend durch den Wechsel von Versorgungsart, -diensten und -akteuren. So beginnt eine Phase im Krankheitsverlauf, die ein höheres $\mathrm{Ma} \beta$ an Autonomie erfordert. Daher übernehmen oft Angehörige eine zentrale Rolle im Versorgungsgeschehen, was auch ihnen viele Anpassungen abverlangt. Dies gelingt umso besser, je gezielter Betroffene und Angehörige darauf vorbereitet werden.

Methode: Es wird ein selbstmanagementförderndes Versorgungsmodell für den Übergang von der stationären in die häusliche Versorgung erprobt, das sich sowohl an die Erkrankten als auch das soziale Umfeld richtet. In der qualitativen und quantitativen Begleitforschung werden u. a. der Nutzen und ,ungeplante Rehospitalisierung' erhoben.

Intervention: Kernelemente sind vier Interventionsmodule: Das Probe-Wohnen beinhaltet alltagsorientierte
Übungs- und Lernmodule zur begleiteten Erprobung von Alltagsabläufen im häuslichen Setting. Beim Training pflegerischer Fertigkeiten wird an einer Pflegepuppe und zwischen Patient/in und Angehörigen körperbezogenes Versorgungshandeln und der Umgang mit Hilfsmitteln geübt. In der Schulung und Kompetenzförderung werden Fähigkeiten zur Bewältigung der veränderten Lebens- und Versorgungssituation vermittelt. Mit dem Selbstinformations-/ Selbstlernzentrum, einer Print- und Online-Bibliothek, wird eine Möglichkeit zur Informationsbeschaffung vor der Entlassung nach Hause bereitgestellt, in deren Rahmen ergänzend die Unterstützung durch Experten aus der stationären Einrichtung in Anspruch genommen werden kann.

Schlussbemerkung: In der Übernahme selbstmanagementfördernder Aufgaben wird eine zentrale Zukunftsaufgabe der studierten Pflege gesehen. Mit einem Erschließen solcher und anderer innovativer Aufgabenfelder wird die Attraktivität des Pflegeberufes erhöht und zur Sicherung des Fachkräftepotenzials beigetragen.

A. Menzel-Begemann $(\bowtie) \cdot$ B. Klünder $\cdot$ D. Schaeffer AG 6: Versorgungsforschung und Pflegewissenschaft, Fakultät für Gesundheitswissenschaften, Universität Bielefeld, Bielefeld, Deutschland

E-Mail: anke.menzel-begemann@uni-bielefeld.de 